\title{
Quantitative hepatitis B surface antigen in predicting recurrence of hepatitis B-related hepatocellular carcinoma after liver transplantation
}

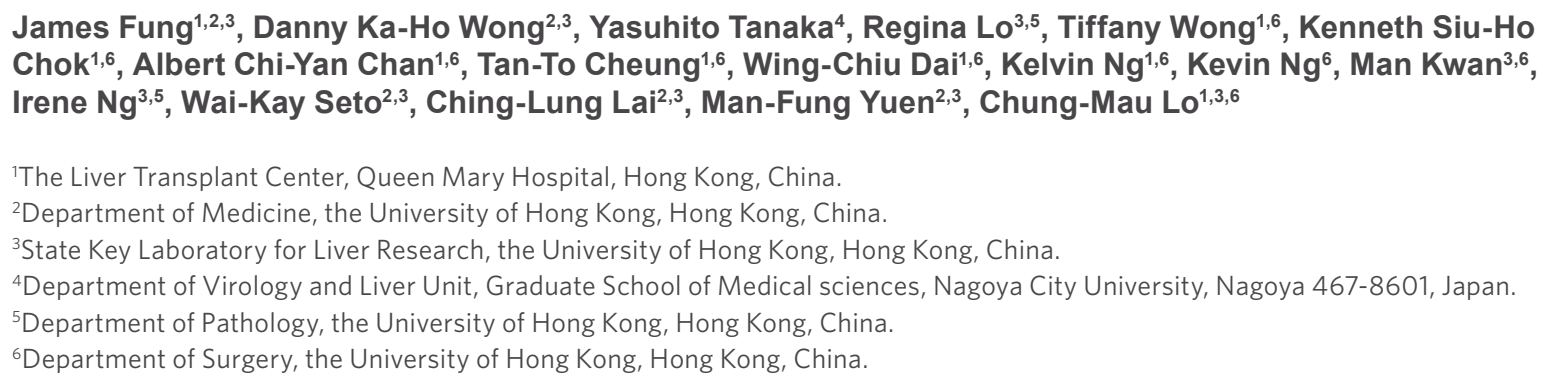

James Fung ${ }^{1,2,3}$, Danny Ka-Ho Wong ${ }^{2,3}$, Yasuhito Tanaka ${ }^{4}$, Regina Lo ${ }^{3,5}$, Tiffany Wong ${ }^{1,6}$, Kenneth Siu-Ho Chok $^{1,6}$, Albert Chi-Yan Chan ${ }^{1,6}$, Tan-To Cheung ${ }^{1,6}$, Wing-Chiu Dai ${ }^{1,6}$, Kelvin Ng ${ }^{1,6}, \mathrm{Kevin} \mathrm{Ng}^{6}$, Man $\mathrm{Kwan}^{3,6}$, Irene $\mathrm{Ng}^{3,5}$, Wai-Kay Seto ${ }^{2,3}$, Ching-Lung Lai ${ }^{2,3}$, Man-Fung Yuen ${ }^{2,3}$, Chung-Mau Lo ${ }^{1,3,6}$

'The Liver Transplant Center, Queen Mary Hospital, Hong Kong, China.

${ }^{2}$ Department of Medicine, the University of Hong Kong, Hong Kong, China.

${ }^{3}$ State Key Laboratory for Liver Research, the University of Hong Kong, Hong Kong, China.

${ }^{4}$ Department of Virology and Liver Unit, Graduate School of Medical sciences, Nagoya City University, Nagoya 467-8601, Japan.

${ }^{5}$ Department of Pathology, the University of Hong Kong, Hong Kong, China.

${ }^{6}$ Department of Surgery, the University of Hong Kong, Hong Kong, China.

Correspondence to: Dr. James Fung, the Liver Transplant Center, Queen Mary Hospital, Hong Kong, China. E-mail: jfung@gastro.hk

How to cite this article: Fung J, Wong DKH, Tanaka Y, Lo R, Wong T, Chok KSH, Chan ACY, Cheung TT, Dai WC, Ng K, Ng K, Kwan M, Ng I, Seto WK, Lai CL, Yuen MF, Lo CM. Quantitative hepatitis B surface antigen in predicting recurrence of hepatitis B-related hepatocellular carcinoma after liver transplantation. Hepatoma Res 2018;4:62. http://dx.doi.org/10.20517/2394-5079.2018.92

Received: 26 Jul 2018 First Decision: 9 Aug 2018 Revised: 30 Aug 2018 Accepted: 30 Aug 2018 Published: 29 Sep 2018

Science Editor: Guang-Wen Cao Copy Editor: Yuan-Li Wang Production Editor: Zhong-Yu Guo

\begin{abstract}
Aim: Recurrence of hepatocellular carcinoma (HCC) after liver transplantation (LT) for chronic hepatitis B (CHB) can be associated with reappearance of hepatitis B surface antigen ( $\mathrm{HBsAg}$ ). The current study determined the significance of $\mathrm{HBsAg}$ qualitatively and quantitatively using a highly sensitive assay in recurrent $\mathrm{HCC}$ after transplantation.
\end{abstract}

Methods: Consecutive patients with HBV-related HCC with LT were included. Oral nucleos(t)ide analogues without hepatitis B immune globulin were used as hepatitis B virus (HBV) prophylaxis. Quantitative HBsAg levels were performed at time of transplant, at 1 month, 3 and 6 months post transplant using a highly sensitive (hs)HBsAg assay.

Results: One hundred and fourteen patients were included, with a median follow-up of 80 months, with 24 cases of $\mathrm{HCC}$ recurrence, and a cumulative rate of $20.7 \%$ at 5 years. There was significant correlation between time of tumor recurrence and time of $\mathrm{HBsAg}$ reappearance $(r=0.551, P=0.027)$. Early HCC recurrence was associated with higher median level of hs-HBsAg at the time of transplant $(72.85 \mathrm{vs} .69 .70 \mathrm{IU} / \mathrm{mL}, P=0.018)$. Using a hs-HBsAg cut-off level of $0.0005 \mathrm{IU} / \mathrm{mL}$, patients with levels above this threshold at 3 and 6 months were

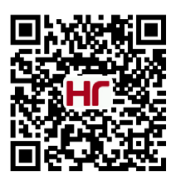


associated with higher rate of early $\mathrm{HCC}$ recurrence $(28.6 \% \mathrm{vs} .3 .0 \%$ and $26.9 \% \mathrm{vs}$. $2.9 \%$ respectively, both $P=$ 0.0006). There was no significant difference in $\mathrm{HCC}$ recurrence between positive and negative $\mathrm{HBsAg}$ using the conventional qualitative $\mathrm{HBsAg}$ assay.

Conclusion: Serum hs-HBsAg levels of $\geq 0.0005 \mathrm{IU} / \mathrm{mL}$ at 3 to 6 months after LT is associated with higher rates of early HCC recurrence, and may be useful as an early tumor marker.

Keywords: Hepatitis B, hepatocellular carcinoma, transplantation, hepatitis B surface antigen, recurrence

\section{INTRODUCTION}

Hepatocellular carcinoma (HCC) is the sixth most common malignancy worldwide, and the third most common cause of cancer death ${ }^{[1]}$. In the majority of cases, HCC develops on the background of chronic liver disease and established cirrhosis. The prevalence of HCC is the highest in the Asia-Pacific region, where chronic hepatitis $\mathrm{B}(\mathrm{CHB})$ infection is the dominant cause ${ }^{[2,3]}$. For many patients, liver transplantation (LT) remains the only curative option. By removing the diseased liver, transplantation potentially cures both the tumour and the underlying cirrhosis. However, not all patients with HCC are eligible for transplantation. The most widely adopted criteria to determine eligibility for transplantation include the Milan and the University of California, San Francisco (UCSF) criteria ${ }^{[4,5]}$. Despite adhering to the selection criteria, there is still a risk of tumour recurrence of approximately $20 \%$ after transplantation ${ }^{[6]}$.

Previous studies have shown different risk factors associated with HCC recurrence after LT, including higher degree of immunosuppression, higher number of tumour nodules, size of the largest lesion, older donor age, presence of vascular invasion, higher pre-operative alpha-fetoprotein (AFP), and higher neutrophil to lymphocyte ratio $^{[7-12]}$. For patients transplanted for hepatitis B virus (HBV)-related HCC, a high pre-operative viral load and inflammatory activity have been shown to be associated with HCC recurrence ${ }^{[13]}$. Other studies have shown an association between HCC and HBV recurrence after $\mathrm{LT}^{[14]}$. A recent study on CHB patients treated with lamivudine monoprophylaxis after LT showed a higher HCC recurrence rate in those who were hepatitis B surface antigen (HBsAg) positive after transplantation ${ }^{[15]}$. Another study demonstrated the detection of HBV DNA and covalently closed circular DNA (cccDNA) in tumour cells, suggesting that HBV replication in tumour cells may contribute to the recurrence of $\mathrm{HBV}^{[16]}$.

The fact that the rate of HCC recurrence in CHB is similar to HCC secondary to other causes after LT suggests that the underlying liver pathology is not a significant predictive factor ${ }^{[17]}$. The association of positive viral markers with HCC recurrence, rather than posing as risk factors per se, may signify these as potential tumour markers in predicting HCC recurrence. The aim of the current study was to determine the significance of HBsAg qualitatively and quantitatively in the recurrence of HCC after LT in patients with CHB.

\section{METHODS}

All patients with HBV-related HCC with LT performed from June 2003 to December 2010 at Queen Mary Hospital (Hong Kong) were included. The selection of patients eligible for LT was carried out using the UCSF criteria (solitary tumour not exceeding $6.5 \mathrm{~cm}$, or a maximum of 3 tumour nodules totaling up to $8 \mathrm{~cm}$ with each nodule not exceeding $4.5 \mathrm{~cm})^{[5]}$. Initial tumour evaluation was performed with triphasic computer tomography (CT) scan of the abdomen and thorax, and with radionuclide bone scan to exclude skeletal metastasis. Dual tracer C11-acetate and 18F-fluorodeoxyglucose positron emission tomography was used in some patients in place of bone scan. Imaging was performed at 3-6 monthly intervals to confirm that the patients remain within the criteria. Bridging loco-regional therapy using transarterial chemoembolization, radiofrequency ablation or high intensity focused ultrasound was offered to patients with a prolonged waiting time. None of the patients received systemic chemotherapy post-transplant. None of the patients had evidence 
of extrahepatic spread or major vascular invasion at the time of transplantation.

\section{Immunosuppression}

The primary immunosuppressive agent used was tacrolimus, with a target therapeutic level of $8-10 \mathrm{ng} / \mathrm{mL}$ in the first 3 months, and a lower range of 5-8 ng/mL beyond 3 months. For patients who were intolerant of tacrolimus, cyclosporine was used. For patients who were intolerant of calcineurin inhibitors or requiring additional immunosuppression, mycophenolate mofetil, sirolimus, and corticosteroids were used. Intravenous hydrocortisone and basiliximab were administered peri-operatively.

\section{Prophylaxis for hepatitis B}

Oral nucleos(t)ide analogues were given as prophylaxis after transplantation according to protocol for prevention of recurrent graft hepatitis. Prior to November 2007, lamivudine was used, with additional rescue therapy for those with evidence of lamivudine resistance. For those with pre-existing lamivudine resistance, lamivudine together with adefovir (and later tenofovir) was given. From November 2007 onwards, entecavir replaced lamivudine as the primary HBV prophylactic agent. As the center adopted an all-oral antiviral regimen, hepatitis B immune globulin (HBIG) was not used in the peri-operative or post-transplant period.

\section{Surveillance for hepatitis B}

Patients were followed up routinely at 3-monthly intervals once stable, or at shorter intervals depending on the clinical need. HBV serology was performed at routine follow up visits, including HBsAg, anti-HBs, and HBV DNA. The qualitative HBsAg tests were performed using the Architect HBsAg assay (Abbott Diagnostics, Abbott Park, IL, USA), with a lower limit of detection (LLOD) of $0.05 \mathrm{IU} / \mathrm{mL}$. Anti-HBs were measured using the Architect anti-HBs assay (Abbott Diagnostics, Abbott Park, IL, USA), with a LLOD of $10 \mathrm{mIU} / \mathrm{mL}$. HBV DNA was measured initially using the Cobas Amplicor assay (Roche Diagnostics, Branchburg, NJ) with a LLOD of 300 copies $/ \mathrm{mL}$, and later with the COBAS Taqman assay (Roche Molecular Systems, Branchburg, NJ) with a LLOD of $20 \mathrm{IU} / \mathrm{mL}$.

To determine the predictive value of HBsAg in early HCC recurrence, quantitative HBsAg levels were performed at the time of transplant, at 1 month, 3 and 6 months post transplant on available stored sera kept at $-20{ }^{\circ} \mathrm{C}$ using a highly sensitive semi-automated immune complex transfer chemiluminescence enzyme immunoassay (ICT-CLEIA) in a HISCL-2000 chemiluminescence immunoassay analyzer (Sysmex, Kobe, Japan ${ }^{[18]}$. Briefly, the samples were incubated with anti-HBs together with magnetic microparticles coated with anti-2,4-dinitorphenol monoclonal antibodies. The samples were then washed and further incubated. The reaction was then performed using the 12GC PLUS Magtration System (Precision System Science, Matsudo, Japan), and the results calculated using an in-house standard curve. The positive results were then confirmed with anti-HBs neutralizing antibodies. This highly sensitive HBsAg (hs-HBsAg) assay had a LLOD of $0.0005 \mathrm{IU} / \mathrm{mL}$.

\section{Surveillance for HCC}

Regular surveillance for HCC was performed after LT with AFP and contrast CT or magnetic resonance imaging (MRI) scans of the abdomen and thorax at 3-6 monthly intervals during the initial 5 years after transplantation and at 6-12 monthly intervals thereafter.

\section{Immunohistochemistry}

Staining for HBsAg was performed on recurrent HCC specimens. The primary antibody HBsAg (clone: S1210, diluted 1:100, Signet) was applied to $4-\mu \mathrm{m}$-thick, $10 \%$ formalin-fixed, paraffin-embedded tissue sections. The sections were first deparaffinized, rehydrated, and washed with xylene, graded alcohol and distilled water. The slides were applied on Leica Bond-III autostainer. No antigen retrieval was required. The specific antibody was located by a linking post primary antibody conjugated to a peroxidase-labeled polymer (biotin-free) that 
Table 1. Patient characteristics

\begin{tabular}{ll}
\hline Parameter & \multicolumn{1}{c}{ Value } \\
\hline Total & 112 \\
Age (years) & $55(30-67)$ \\
Gender & \\
$\quad$ Males & $97(86.6 \%)$ \\
Females & $15(13.2 \%)$ \\
Follow-up length (months) & $80.5(2-145)$ \\
Type of transplant & \\
Living donor & $80(71.4 \%)$ \\
Deceased donor & $32(28.6 \%)$ \\
Tumour characteristics & \\
Tumour size (cm) & $3(1-8)$ \\
Tumour number & $1(1-15)$ \\
Alpha-fetoprotein (ng/mL) & $18(1-33858)$ \\
Viral parameters & \\
Hepatitis B e-antigen positive & $35(31.3 \%)$ \\
HBV DNA (log IU/mL) & $2.52(1.54-9.75)$ \\
HBV DNA undetectability & $44(39.3 \%)$ \\
Hepatitis B surface antigen (IU/mL) & $70.88(0.0009-75.91)$ \\
Antiviral therapy at transplantation & \\
Lamivudine & $51(45.5 \%)$ \\
Entecavir & $46(41.1 \%)$ \\
Lamivudine + adefovir & $10(8.9 \%)$ \\
Lamivudine + tenofovir & $4(3.6 \%)$ \\
Entecavir + adefovir & $1(0.9 \%)$ \\
\hline & \\
Continuous variables expressed as median vales (range) &
\end{tabular}

recognized mouse and rabbit immunoglobulins. Hydrogen peroxidase was applied to remove endogenous peroxidase activity. The polymer complex was then visualized with an appropriate diaminobenzidine (DAB) chromogen. The sections were counterstained with hematoxylin. Appropriate positive and negative controls were used.

Informed consent was obtained from all patients for collection and storage of clinical specimens for use in the current project, and approved by the Ethics Committee Institutional Review Board of the University of Hong Kong/Hospital Authority Hong Kong Western Cluster (UW 05-359 T/1022).

\section{Statistical analysis}

All statistical analyses were performed using the SPSS version 17.0 (SPSS Inc, Chicago, IL). Categorical variables were analyzed using the Chi-squared test, and Fisher's exact test when appropriate. Mann-Whitney test was used to analyze continuous variables with skewed distribution, and Kruskal-Wallis test used for continuous variables with more than 2 categories. Bivariate correlation for continuous variables was performed using the Pearson test. The cumulative incidences of HCC recurrences and survivals were analyzed using the Kaplan-Meier method, with log-rank testing for comparison. A $P$-value of $<0.05$ was considered statistically significant.

\section{RESULTS}

A total of 114 consecutive patients were transplanted for HBV-related HCC from June 2003 to December 2010. Of the 114 patients, 2 patients did not have HBsAg determined after transplantation due to early mortality from cardiac arrest, leaving 112 in the final analysis. The median follow-up was 80.5 months (range, 2 to 145 ), with a median age of 55 years (range 30-67) and a male predominance (86.6\%). Of the 112 patients, 80 (71.4\%) and $32(28.6 \%)$ underwent living-related and deceased-donor LT respectively. The patient characteristics, tumour characteristics, and the types of antiviral regimen used at the time of transplantation are summarized in Table 1. The tumour size and number were based on explant histology. At the time of transplant, 31.3\% were Hepatitis Be Antigen (HBeAg) positive and the median HBV DNA and HBsAg level was $2.52 \log \mathrm{IU} / \mathrm{mL}$ and $70.88 \mathrm{IU} / \mathrm{mL}$ respectively. There was no correlation between pre-transplant HBsAg levels and HBV DNA 
$(P=0.451)$ or AFP $(P=0.402)$. There was no difference in pre-transplant HBsAg levels between the numbers of HCC nodules (1-3; $P=0.620$ ), and HCC differentiation (well, moderate, poor; $P=0.740$ ).

\section{Post transplant hepatitis B status}

The majority of patients underwent loss of HBsAg shortly after transplantation, with a cumulative rate of HBsAg seroclearance of $90.7 \%$ at 6 months after transplantation [Figure 1A]. Only 5 patients remained persistently positive for HBsAg after transplantation without evidence of seroclearance. A total of 27 patients had re-appearance of HBsAg after initial HBsAg seroclearance after transplantation. The cumulative rate of HBsAg re-appearance was $8.5 \%, 18.0 \%, 21.9 \%$, and $26.4 \%$ at $1,2,3$, and 5 years respectively, with no further increase thereafter. There was no difference between living-related and deceased-donor LT with respect to reappearance of HBsAg $(P=0.945)$.

Despite the absence of HBIG administration, 64 (57.1\%) of the patients had detectable anti-HBs titer after transplantation [Figure $1 \mathrm{~B}]$. The majority occurred within the early post transplant period, with a median time to antibody development of 2 months (range, 0-137), and a median peak antibody level of $133 \mathrm{mIU} /$ $\mathrm{mL}$ (range, 11 to $>1000$ ). The detectable antibody titres represented a transient phenomenon as $56(87.5 \%)$ had subsequent disappearance of antibodies with a median time of 7 months from the time of antibody appearance (range, 0 to 131).

All patients achieved undetectable HBV DNA post transplant, with a cumulative rate of undetectable HBV DNA of $97.3 \%$ at 6 months. Virological rebound was defined as a $1 \log$ increase from nadir. The cumulative rate of virological rebound was $20.5 \%$ and $31.0 \%$ at 5 and 10 years after transplantation respectively. A total of 28 patients had evidence of virological rebound, of which 5 had no evidence of rtM204 mutation, and 6 with pre-existing rtM204 mutation. The remaining 17 patients had newly detected rtM204 mutation, and all were treated with additional nucleos $(\mathrm{t})$ ide analog therapy.

\section{Overall recurrence of $\mathrm{HCC}$}

There were 24 cases of HCC recurrence during the follow-up period. The overall cumulative rate of HCC recurrence was $9.0 \%, 14.4 \%, 20.7 \%$, and $24.3 \%$ at 1,3 , 5, and 10 years respectively [Figure $1 \mathrm{C}$ ]. The AFP at the time of LT was higher for those with early HCC recurrence compared to those without (38vs. $14 \mathrm{ng} / \mathrm{mL}$ respectively, $P=0.027)$. The sensitivity of an elevated AFP $(>20 \mathrm{ng} / \mathrm{mL})$ at $1,3,6$, and 12 months in diagnosing early HCC recurrence, as defined by recurrence within 3 years after transplantation, was $8 \%, 7 \%, 21 \%$, and $20 \%$ respectively. There was no difference in tumour size between those with and without early recurrence $(P=0.835)$. The median number of HCCs on explant was higher for those with early recurrence ( 3 vs. $1, P<0.001)$.

\section{HBV DNA levels with HCC recurrence}

There was no difference in the HBV DNA levels at the time of transplantation between those with and without early HCC recurrence (both groups had median HBV DNA levels at the LLOD, $P=0.231$ ). There was no significant differences in the rate of early HCC recurrence between those with and without undetectable HBV DNA levels at $1,3,6$, and 12 months after transplantation $(P=0.448, P=0.579, P=0.308$, and $P=0.608$ respectively).

\section{HBsAg status and $\mathrm{HCC}$ recurrence}

There was significantly lower HCC recurrence rates for those with persistent loss of HBsAg compared to those that remained HBsAg positive ( $7 \%$ vs. $40.0 \%$ respectively at 5 years post transplant, $P=0.012$ ). Twentyseven patients had re-appearance of HBsAg after initial HBsAg seroclearance. For those with evidence of HBsAg seroclearance, re-appearance of HBsAg was associated with a significantly higher rate of HCC recurrence compared to those who remained HBsAg negative $(56.1 \%$ vs. $7 \%$ respectively at 5 years post transplant, $P<0.001$ ) [Figure $2 \mathrm{~A}$ ]. 
A

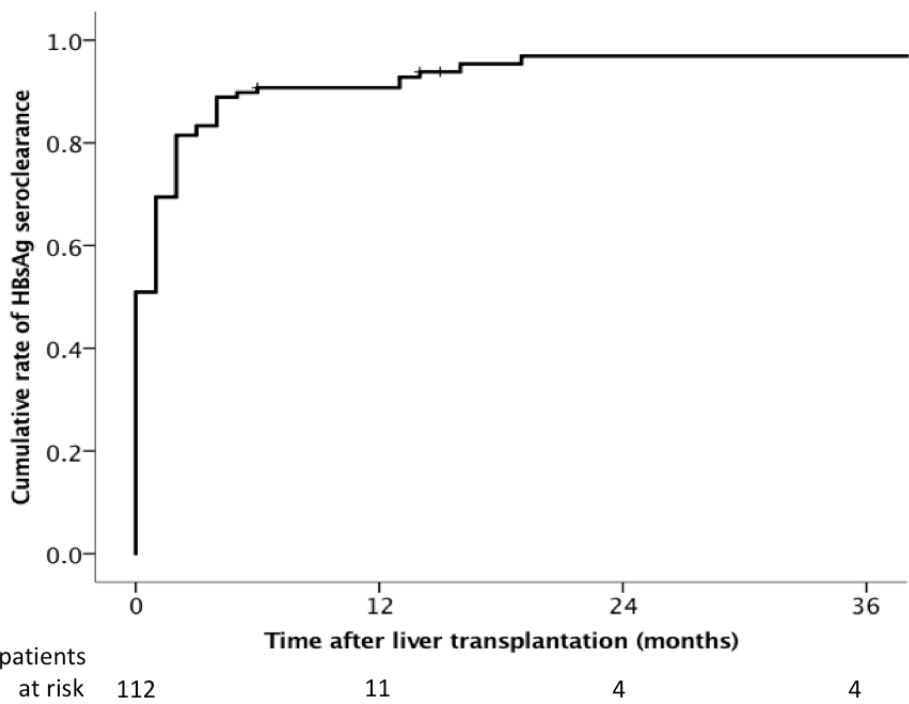

\section{B}

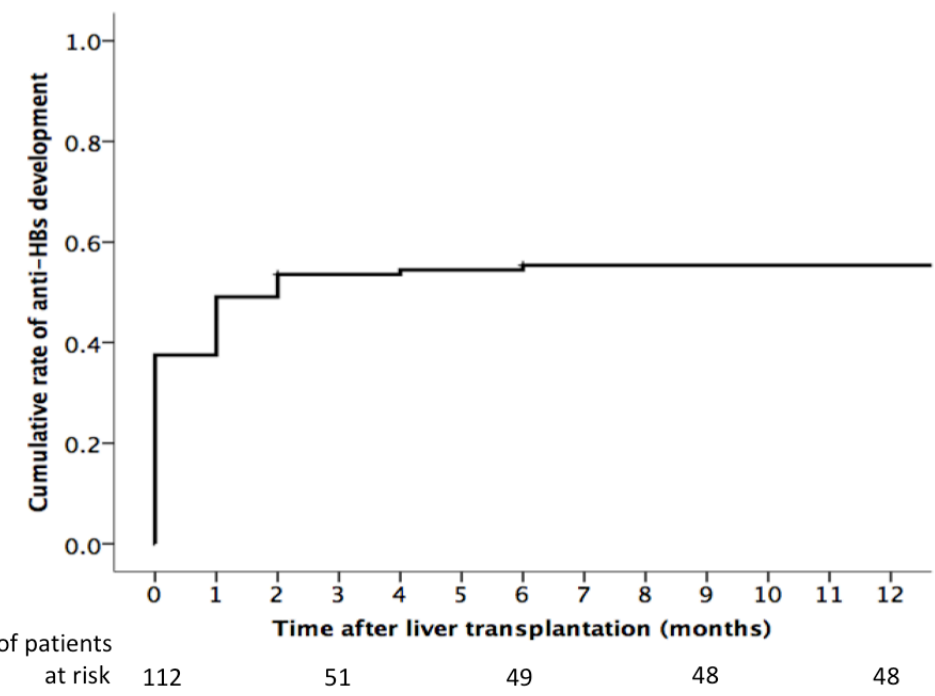

C

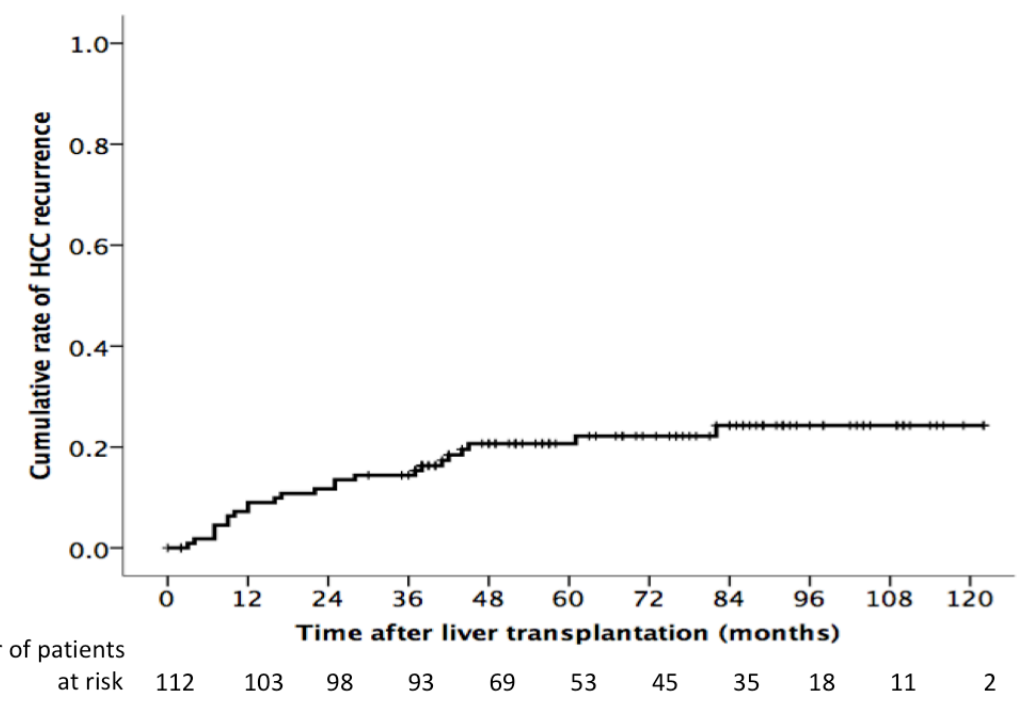

Figure 1. A: Cumulative incidence of $\mathrm{HBsAg}$ seroclearance after liver transplantation; B: cumulative incidence of the development of detectable anti-HBs after liver transplantation; C: cumulative incidence of recurrent hepatocellular carcinoma after transplantation 
A

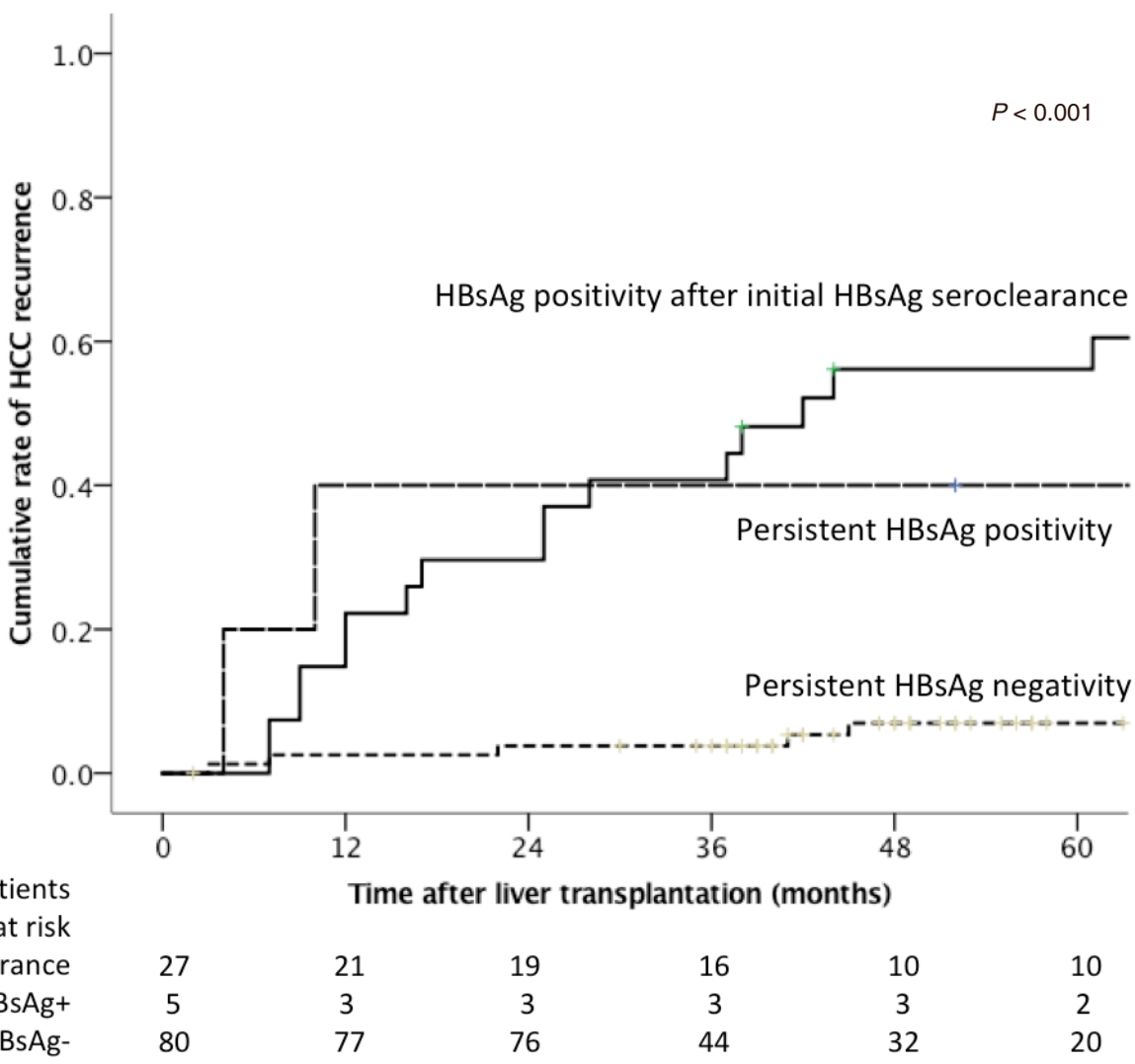

B

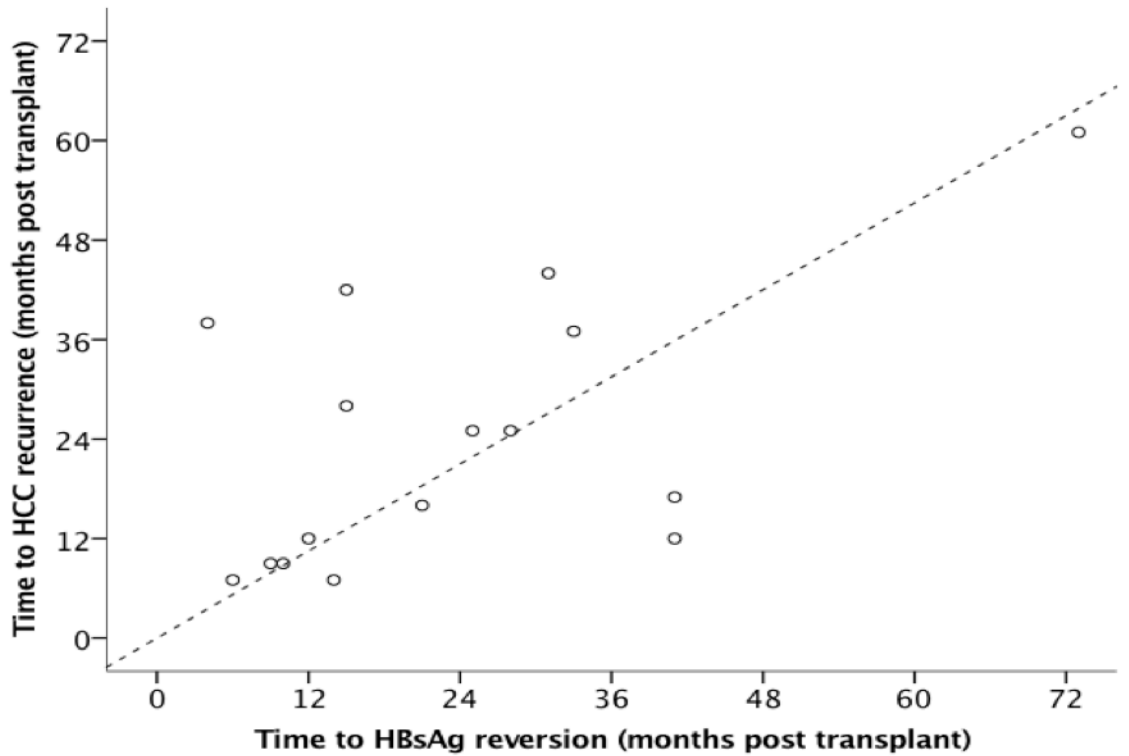

Figure 2. A: Cumulative incidence of recurrent hepatocellular carcinoma according to HBsAg status after liver transplantation; B: correlation between time of hepatocellular carcinoma recurrence and $\mathrm{HBs} A g$ seroreversion after liver transplantation 


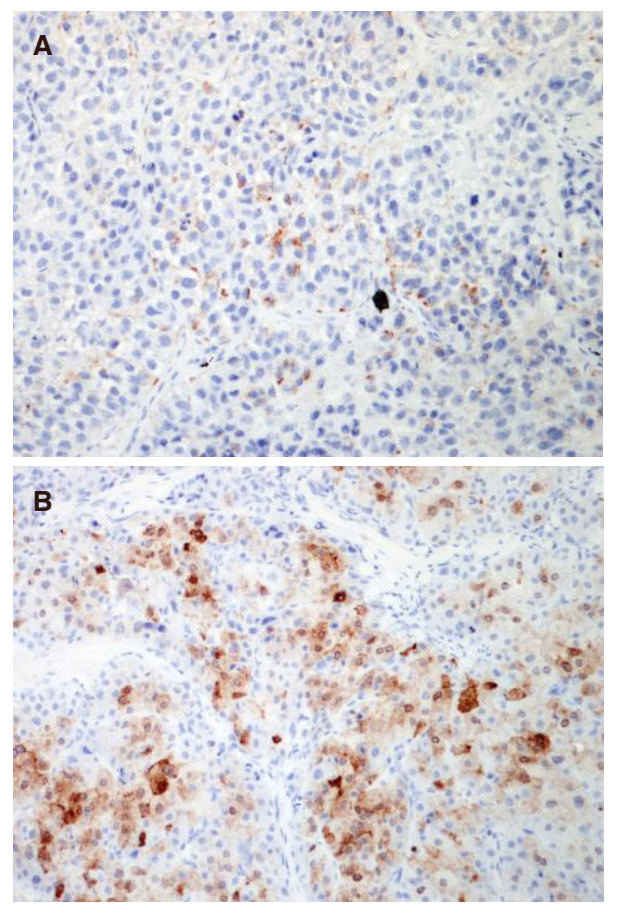

Figure 3. A and B: Immunohistochemical study for $\mathrm{HBsAg}$ showing positive cytoplasmic staining in metastatic tumour cells in lungs

A total of 16 patients lost HBsAg after transplantation, with subsequent re-appearance of HBsAg and recurrence of HCC. There was a significant correlation between the time of tumour recurrence and the time of HBsAg re-appearance $(r=0.551, P=0.027)$, as shown in Figure 2B. There was no significant difference in the median time of recurrence of HCC vs. reappearance of HBsAg after LT (21 months vs. 18 months respectively, $P=0.809$ ). Of these 16 patients, 2 had recurrence limited to the liver, and the remaining 14 patients had extra-hepatic metastatic lesions to the lungs, bones, lymph nodes, and adrenal glands. Histology from the site of recurrence was available for 12 of 16 patients, with specimens from 2 patients staining positive for HBsAg (both from metastatic lung tissues) [Figure 3]. One had recurrence at 9 months after transplantation, with HBsAg re-appearance at 10 months. The other patient had recurrence at 7 months, with HBsAg re-appearance at 14 months.

\section{Quantitative HBsAg levels with HCC recurrence}

There was a significant higher median level of hs-HBsAg at the time of transplant for those with early HCC recurrence compared to those without (72.85 vs. $69.70 \mathrm{IU} / \mathrm{mL}$ respectively, $P=0.018)$. After transplant, the median hs-HBsAg levels at month 1, 3, 6, and 12 was 0.0008 (range, 0-50.6855), 0 (range, 0-1.0827), 0 (range, $0-0.1642$ ), and 0 (range, 0-0.1310) IU/mL respectively. Using a hs-HBsAg cut-off level of $0.0005 \mathrm{IU} / \mathrm{mL}$, patients with levels $\geq 0.0005 \mathrm{IU} / \mathrm{mL}$ was associated with a significantly higher rate of early HCC recurrence compared to those with lower levels at 3 months post transplant $(28.6 \% v$ s. $3.0 \%$ respectively at 3 years post transplant, $P=0.006)$ [Figure $4 \mathrm{~A}$ ], and at 6 months post transplant $(26.9 \%$ vs. $2.9 \%$ respectively, $P=0.006)$ [Figure $4 \mathrm{~B}$ ]. In contrast, using the conventional qualitative HBsAg assay, there was no significant difference in HCC recurrence observed between positive and negative HBsAg status at 3 and 6 months post transplant $(P=0.845$ and $P=0.449$, respectively). No significant difference in early HCC recurrence rate was observed at 1 month post transplant using this cut-off $(P=0.162)$ [Figure $4 \mathrm{C}$ ].

\section{DISCUSSION}

In $\mathrm{CHB}$ patients who achieve $\mathrm{HBsAg}$ seroclearance by conventional assays, a substantial proportion of 

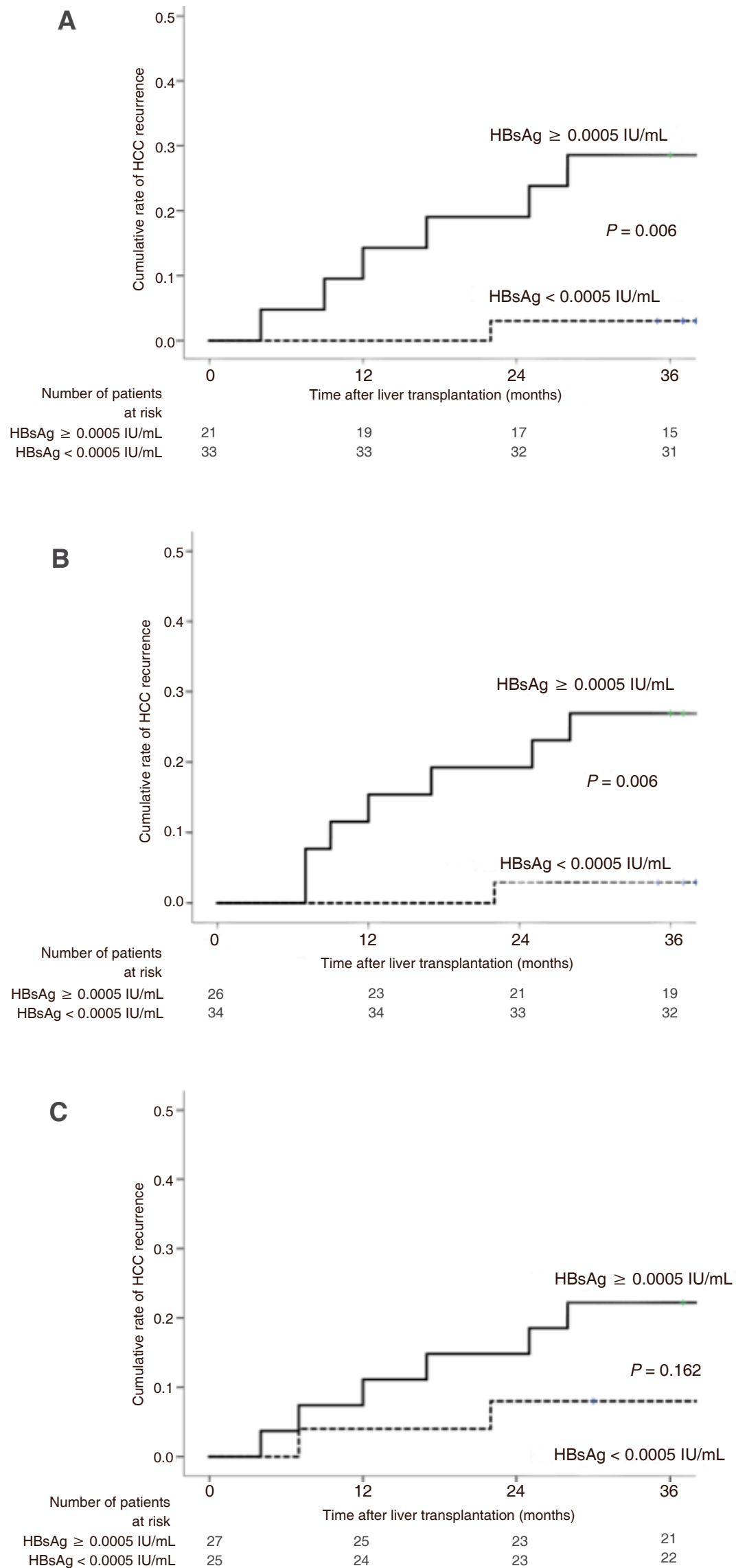

Figure 4. A: Cumulative incidence of hepatocellular carcinoma recurrence according to hs-HBsAg level at 3 months after liver transplantation; B: cumulative incidence of hepatocellular carcinoma recurrence according to hs-HBsAg level at 6 months after liver transplantation; C: cumulative incidence of hepatocellular carcinoma recurrence according to hs-HBsAg level at 1 month after liver transplantation 
patients may show detectable HBsAg using the hs-HBsAg assay, especially those who were negative for anti-HBs ${ }^{[19]}$. This would suggest that HBsAg seroclearance only implies undetectability below the standard quantitative assay. For the first time, the current study demonstrated that quantitative HBsAg levels using a highly sensitive assay could be used to predict higher chance of HCC recurrence after LT for HBV-related HCC. By using a completely HBIG-free regimen for hepatitis B prophylaxis, the study population presented a unique opportunity to study the characteristics of HBsAg both qualitatively and quantitatively after transplantation, and its association with early HCC recurrence. The administration of HBIG will preclude any useful determination of HBsAg, as these will largely become undetectable through binding to anti-HBs, which is consistently kept at a high level through the use of regular injections. Oral nucleos(t)ide analogues, although effective in suppressive HBV DNA to undetectable levels, has significantly less effect on the HBsAg levels. Previous studies have demonstrated that even potent oral antiviral therapy may not reduce HBsAg levels despite prolonged HBV DNA suppression to undetectable levels ${ }^{[20]}$.

The current study showed a significantly higher rate of HCC recurrence in those who failed to achieve HBsAg seroclearance after transplantation (50.0\% vs. 23.4\% respectively at 8 years post transplant, $P=0.024$ ). Furthermore, for patients who remained HBsAg positive after transplant, the recurrence of HCC was early (within the first year of transplant). It is possible that micrometastasis present at the time of transplant may be responsible for the persistence of HBsAg. In addition, a significantly higher HCC recurrence rate was observed in those who had HBsAg seroreversion compared to those who remained negative for HBsAg (60.5\% vs. 9.4\% respectively at 10 years post transplant, $P<0.001$ ). The association of recurrence HCC and HBV have also been described in a smaller cohort of patients previously ${ }^{[21]}$. Since extra-hepatic tumour tissue stained positive for HBsAg, it is likely that malignant tumour cells are able to support HBsAg production. Moreover, there was correlation between the timing of HBsAg re-appearance and HCC recurrence $(r=0.551, P=0.027)$, raising the possibility that HBsAg may be useful as a marker of HCC recurrence. The temporal relationship suggests that the HBsAg is unlikely to have a role in the pathogenesis of HCC recurrence, but rather HCC may be responsible for the HBsAg reversion.

To date, the only tumour marker currently readily available for post transplant HCC recurrence is serum AFP, which in the current study, showed low sensitivity (7\% to $21 \%$ ) as an early predictor after transplantation. In addition, a significant proportion of HCC did not secrete AFP. The finding that hs-HBsAg levels above the LLOD ( $\geq 0.0005 \mathrm{IU} / \mathrm{mL}$ ) at 3 and 6 months can be associated with higher rates of early HCC recurrence suggests that this may be used as an early tumour marker. In contrast, the less sensitive conventional HBsAg measurement did not show a significant difference at 3 or 6 months post transplant with respect to a positive or negative HBsAg status and subsequent HCC recurrence. This may be due to the fact that during the early post transplant period, tumour load is likely to be extremely low for those with recurrence, and therefore the HBV DNA may not be detectable and HBsAg may not be quantifiable by conventional assays.

One of the consistent observations with HBIG-free prophylaxis after transplantation is the development of detectable transient levels of anti-HBs titers shortly after transplantation, despite the absence of HBIG administration (including the current study) ${ }^{[22-24]}$. This is most likely due to passive transfer of antibodies from the donor, and is largely non-sustainable. Importantly, this phenomenon may preclude the use of quantitative HBsAg as a useful tool for predicting HCC recurrence in the very early phases (within 3 months) after transplantation, and provide explanation as to why the HBsAg levels were not predictive at 1-month post transplant period in the current study. Other intermediate replication markers such as the hepatitis B corerelated antigen may be more useful, and deserves further study.

There are several limitations to the current study. Firstly, quantitative hs-HBsAg was performed in available stored samples only. A previous study looking at the change in HBsAg levels with the conventional assay in stored sera found no significant changes over time ${ }^{[25]}$. Secondly, this cohort consists of patients largely in the 
lamivudine era, and hence accounts for the high rate of virological rebound due to the development of rtM204 mutations. The virological rebound observed would likely have impact on the predictive value of quantitative HBsAg in predicting HCC recurrence. Thirdly, there was a lower proportion with deceased-donor LT with a lower recurrence rate, thus precluding any comparison with those undergoing living-donor LT.

To conclude, serum hs-HBsAg levels of $\geq 0.0005 \mathrm{IU} / \mathrm{mL}$ at 3-6 months after LT have been shown to be associated with higher rates of early HCC recurrence, and may be a useful tool as an early tumour marker in the post transplant setting.

\section{DECLARATIONS}

\section{Acknowledgements}

Dr. Shintaro Oagawa exams the hs-HBsAg assay.

\section{Authors' contributions}

Planning and conduction of study, collecting and interpreting data, drafting manuscript: Fung J

Conduction of study, laboratory supervision and work, critical appraisal: Wong DKH

Laboratory work, critical appraisal: Tanaka Y, Ng K

Laboratory work, collecting and interpreting data, critical appraisal: Lo R

Conduction of study, collecting data, critical appraisal: Wong T, Chok KSH, Chan ACY, Cheung TT, Dai WC, $\mathrm{Ng} \mathrm{K}$

Laboratory supervision and work, critical appraisal: Kwan M, Ng I

Conduction of study, critical appraisal: Seto WK

Design of study and critical appraisal: Lai CL

Planning \& design of study, critical appraisal, and study supervision: Yuen MF

Critical appraisal and study supervision: Lo CM

\section{Availability of data and materials}

On request.

\section{Financial support and sponsorship}

None.

\section{Conflicts of interest}

All authors declared that there are no conflicts of interest.

\section{Ethical approval and consent to participate}

This study has been performed in accordance with the Declaration of Helsinki. Informed consent was obtained from all patients for collection and storage of clinical specimens for use in the current project, and approved by the Ethics Committee Institutional Review Board of the University of Hong Kong/Hospital Authority Hong Kong Western Cluster (UW 05-359 T/1022).

\section{Consent for publication}

Not applicable.

\section{Copyright}

(c) The Author(s) 2018.

\section{REFERENCES}

1. Ferlay J, Shin HR, Bray F, Forman D, Mathers C, Parkin DM. Estimates of worldwide burden of cancer in 2008: GLOBOCAN 2008. Int J 
Cancer 2010;127:2893-917.

2. Sherman M. Hepatocellular carcinoma: epidemiology, surveillance, and diagnosis. Semin Liver Dis 2010;30:3-16.

3. Yuen MF, Hou JL, Chutaputti A; Asia Pacific Working Party on Prevention of Hepatocellular Carcinoma. Hepatocellular carcinoma in the Asia pacific region. J Gastroenterol Hepatol 2009;24:346-53.

4. Mazzaferro V, Regalia E, Doci R, Andreola S, Pulvirenti A, Bozzetti F, Montalto F, Ammatuna M, Morabito A, Gennari L. Liver transplantation for the treatment of small hepatocellular carcinomas in patients with cirrhosis. N Engl J Med 1996;334:693-9.

5. Yao FY, Ferrell L, Bass NM, Watson JJ, Bacchetti P, Venook A, Ascher NL, Roberts JP. Liver transplantation for hepatocellular carcinoma: expansion of the tumor size limits does not adversely impact survival. Hepatology 2001;33:1394-403.

6. Welker MW, Bechstein WO, Zeuzem S, Trojan J. Recurrent hepatocellular carcinoma after liver transplantation - an emerging clinical challenge. Transpl Int 2013;26:109-18.

7. Sharma P, Welch K, Hussain H, Pelletier SJ, Fontana RJ, Marrero J, Merion RM. Incidence and risk factors of hepatocellular carcinoma recurrence after liver transplantation in the MELD era. Dig Dis Sci 2012;57:806-12.

8. Han SH, Reddy KR, Keeffe EB, Soldevila-Pico C, Gish R, Chung RT, Degertekin B, Lok A; NIH HBV OLT Study Group. Clinical outcomes of liver transplantation for HBV-related hepatocellular carcinoma: data from the NIH HBV OLT study. Clin Transplant 2011;25:E152-62.

9. Wu JC, Huang YH, Chau GY, Su CW, Lai CR, Lee PC, Huo TI, Sheen IJ, Lee SD, Lui WY. Risk factors for early and late recurrence in hepatitis B-related hepatocellular carcinoma. J Hepatol 2009;51:890-7.

10. Vivarelli M, Bellusci R, Cucchetti A, Cavrini G, De Ruvo N, Aden AA, La Barba G, Brillanti S, Cavallari A. Low recurrence rate of hepatocellular carcinoma after liver transplantation: better patient selection or lower immunosuppression? Transplantation 2002;74:1746-51.

11. Wang GY, Yang Y, Li H, Zhang J, Jiang N, Li MR, Zhu HB, Zhang Q, Chen GH. A scoring model based on neutrophil to lymphocyte ratio predicts recurrence of HBV-associated hepatocellular carcinoma after liver transplantation. PLoS One 2011;6:e25295.

12. Shimoda M, Ghobrial RM, Carmody IC, Anselmo DM, Farmer DG, Yersiz H, Chen P, Dawson S, Durazo F, Han S, Goldstein LI, Saab S, Hiatt J, Busuttil RW. Predictors of survival after liver transplantation for hepatocellular carcinoma associated with Hepatitis C. Liver Transpl 2004;10:1478-86.

13. Li MR, Chen GH, Cai CJ, Wang GY, Zhao H. High hepatitis B virus DNA level in serum before liver transplantation increases the risk of hepatocellular carcinoma recurrence. Digestion 2011;84:134-41.

14. Saab S, Yeganeh M, Nguyen K, Durazo F, Han S, Yersiz H, Farmer DG, Goldstein LI, Tong MJ, Busuttil RW. Recurrence of hepatocellular carcinoma and hepatitis B reinfection in hepatitis B surface antigen-positive patients after liver transplantation. Liver Transpl 2009;15:152534.

15. Fung J, Chan SC, Yuen MF, Lai CL, Chok KSH, Sharr W, Dai WC, Fan ST, Lo CM. Long-term outcome of lamivudine mono-prophylaxis after liver transplantation in hepatitis B patients. Hepatology 2011;54:450A.

16. Faria LC, Gigou M, Roque-Afonso AM, Sebagh M, Roche B, Fallot G, Ferrari TC, Guettier C, Dussaix E, Castaing D, Brechot C, Samuel D. Hepatocellular carcinoma is associated with an increased risk of hepatitis B virus recurrence after liver transplantation. Gastroenterology 2008;134:1890-9.

17. Bozorgzadeh A, Orloff M, Abt P, Tsoulfas G, Younan D, Kashyap R, Jain A, Mantry P, Maliakkal B, Khorana A, Schwartz S. Survival outcomes in liver transplantation for hepatocellular carcinoma, comparing impact of hepatitis $\mathrm{C}$ versus other etiology of cirrhosis. Liver Transpl 2007;13:807-13.

18. Takeda K, Maruki M, Yamagaito T, Muramatsu M, Sakai Y, Tobimatsu H, Kobayashi H, Mizuno Y, Hamaguchi Y. Highly sensitive detection of hepatitis B virus surface antigen by use of a semiautomated immune complex transfer chemiluminescence enzyme immunoassay. J Clin Microbiol 2013;51:2238-44

19. Seto WK, Tanaka Y, Wong DK, Shinkai N, Cheung KS, Liu KS, Fung J, Lai CL, Yuen MF. Longitudinal profiles of highly sensitive hepatitis B surface antigen levels: re-evaluation of HBsAg seroclearance. Liver Int 2016;36:642-50.

20. Fung J, Lai CL, Young J, Wong DK, Yuen J, Seto WK, Yuen MF. Quantitative hepatitis B surface antigen levels in patients with chronic hepatitis B after 2 years of entecavir treatment. Am J Gastroenterol 2011;106:1766-73.

21. Kiyici M, Yilmaz M, Akyildiz M, Arikan C, Aydin U, Sigirli D, Nart D, Yilmaz F, Ozacar T, Karasu Z, Kilic M. Association between hepatitis B and hepatocellular carcinoma recurrence in patients undergoing liver transplantation. Transplant Proc 2008;40:1511-7.

22. Fung J, Cheung C, Chan SC, Yuen MF, Chok KS, Sharr W, Dai WC, Chan AC, Cheung TT, Tsang S, Lam B, Lai CL, Lo CM. Entecavir monotherapy is effective in suppressing hepatitis B virus after liver transplantation. Gastroenterology 2011;141:1212-9.

23. Fung J, Chan SC, Cheung C, Yuen MF, Chok KS, Sharr W, Chan AC, Cheung TT, Seto WK, Fan ST, Lai CL, Lo CM. Oral nucleoside/ nucleotide analogs without hepatitis B immune globulin after liver transplantation for hepatitis B. Am J Gastroenterol 2013;108:942-8.

24. Lo CM, Fung JT, Lau GK, Liu CL, Cheung ST, Lai CL, Fan ST, Wong J. Development of antibody to hepatitis B surface antigen after liver transplantation for chronic hepatitis B. Hepatology 2003;37:36-43.

25. Fung J, Lai CL, Young J, Wong DK, Yuen J, Seto WK, Yuen MF. Stability of hepatitis B surface antigen over time: implications for studies using stored sera. J Med Virol 2011;83:1900-4. 